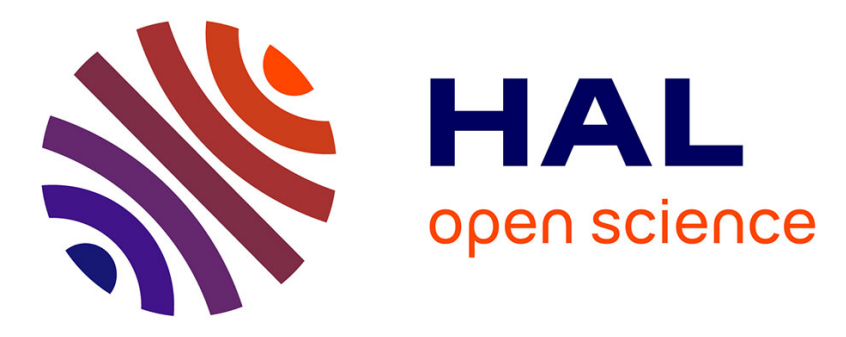

\title{
Deux témoins d'Ambroise sur le psaume 118 et leur ancêtre
}

\author{
Pierre Chambert-Protat
}

\section{To cite this version:}

Pierre Chambert-Protat. Deux témoins d'Ambroise sur le psaume 118 et leur ancêtre. Mariken Teeuwen; Irene van Renswoude. The Annotated Book in the Early Middle Ages: Practices of Reading and Writing, 38, Brepols, pp.647-672, 2017, Utrecht Studies in Medieval Literacy, 978-2-503-56948-2. 10.1484/M.USML-EB.5.115037 . halshs-01631460

\section{HAL Id: halshs-01631460 \\ https://shs.hal.science/halshs-01631460}

Submitted on 30 Mar 2018

HAL is a multi-disciplinary open access archive for the deposit and dissemination of scientific research documents, whether they are published or not. The documents may come from teaching and research institutions in France or abroad, or from public or private research centers.
L'archive ouverte pluridisciplinaire HAL, est destinée au dépôt et à la diffusion de documents scientifiques de niveau recherche, publiés ou non, émanant des établissements d'enseignement et de recherche français ou étrangers, des laboratoires publics ou privés. 


\title{
Deux témoins d'Ambroise sur le Psaume 118 et leur ancêtre
}

\author{
Pierre CHAMBERT-PROTAT \\ École française de Rome
}

ORCID $\underline{0000-0002-9992-2683}$

Ce document est la version d'auteur d'une contribution parue dans

M.J. TeEuWEn et I. van Renswoude (dirs.), The Annotated Book

in the Early Middle Ages : Practices of Reading and Writing, Turnhout :

Brepols, 2017 (Utrecht Studies in Medieval Literacy, 38), pp. 647-672.

DOI : $\underline{10.1484 / M . U S M L-E B .5 .115037}$; halshs-01631460

La présente étude porte sur deux manuscrits, FIRENZE, BML, Plut. XIV. 21 et PARIS, BNF, n.a.l. 1437, dont les datation et origine seront précisées plus loin. Ils sont tous deux témoins d'un même texte, le volumineux commentaire d'Ambroise de Milan sur le psaume 118 (AMBR. in psalm. 118 serm.), et pourtant leurs rapports phylogénétiques n'ont pas été bien démêlés jusqu'à présent. Afin de comprendre pourquoi, il importe de refaire d'abord l'état de la question en reprenant la bibliographie dans l'ordre.

\section{État de la question}

\section{3 : l'édition Petschenig}

Les deux manuscrits étaient connus du dernier éditeur du commentaire, Michael Petschenig ${ }^{1}$, mais ils n'avaient pas pour lui la même importance, loin $\stackrel{648}{=}$ 'en faut. Avec raison, il

1. M. Petschenig (éd.), Sancti Ambrosii Opera. Pars V: Expositio Psalmi CXVIII, Wien, 1913 (Corpus Scriptorum Ecclesiasticorum Latinorum, 62). Une réimpression augmentée d'indices a paru en 1999 (CSEL $62^{\text {bis }}$ : Michaela ZeLZER éd.). 
P. Chambert-Protat, « Deux témoins d'Ambroise sur le Psaume 118 et leur ancêtre », 2017

DOI 10.1484/M.USML-EB.5.115037 ; halshs-01631460

assigne à PARIS n.a.1. 1437 cette origine : « saec. X, olim Cluniacensis $40^{2}$ ». C'est un des témoins les plus importants de l'œuvre, d'autant qu'il est des plus anciens, et presque complet : il ne manque que son dernier feuillet. Petschenig retient donc son témoignage, et le sigle $\mathbf{P}$ dans son apparat.

Il n'ignore pas le Plut. XIV. 21, mais il le date du XI siècle. En le collationnant jusqu'à la seconde «lettre» du commentaire ambrosien ${ }^{3}$, il constate sa parenté étroite avec PARIS n.a.l. 1437 : il écarte donc son témoignage ${ }^{4}$. Ainsi, même s'il ne le dit nulle part explicitement et si, jugeant les familles confuses, il n'a pas livré de stemma, toute sa démarche implique que pour lui le Plut. XIV. 21 est un descendant de PARIs n.a.l. 1437.

Ce diagnostic eut pour conséquence de faire tomber le Plut. XIV. 21 dans l'oubli. Jusqu'en 1998, personne ne put soupçonner l'intérêt de ce qui passait pour un descriptus d'un bon témoin. En revanche, dans cet intervalle, plusieurs recherches ont alimenté et précisé l'histoire du manuscrit clunisien PARIS n.a.l. 1437.

\section{2 : l'étude de Charlier}

Quatre décennies après l'édition de Petschenig, l'inventeur des «manuscrits personnels de Florus de Lyon » et de sa méthode de travail, Célestin Charlier ${ }^{5}$, consacre une importante étude au codex MontPellier, B.U. Fac. Médecine, H. 308, dans lequel il découvre une volumineuse collection patristique et canonique compilée par Florus ${ }^{6}$. Il l'intitule «collection De fide» (désormais FLOR. LVGD. fid.), parce qu'elle commente le Credo et parce que $\stackrel{649}{l}$ 'on trouve, dans certains manuscrits lyonnais, des dispositifs d'excerption accompagnés de sigles marginaux D'F' qui correspondent systématiquement à des extraits compilés dans la collection. La méthode est

2. CSEL 62, p. VIII.

3. Après une courte préface, le commentaire ambrosien suit le découpage du psaume 118, lui-même composé de vingt-deux huitains, un pour chaque lettre de l'alphabet hébreu.

4. « usque ad tractat. 2 collatus prope accedit ad $\mathrm{P} »\left(C S E L\right.$ 62, p. X). C'est son témoin $\mathrm{n}^{\circ} 10$, non siglé.

5. C. CHARLIER, «Les manuscrits personnels de Florus de Lyon et son activité littéraire », dans Mélanges Emmanuel Podechard. Études de sciences religieuses offertes pour son éméritat au doyen bonoraire de la faculté de théologie de Lyon, Lyon, 1945, p. 71-84; ID., «La Compilation augustinienne de Florus sur l'Apôtre : sources et authenticité », dans Revue bénédictine 57 (1947), p. 132-186.

6. C. CharliER, «Une œuvre inconnue de Florus de Lyon : la collection De fide de Montpellier» dans Traditio 8 (1952), p. 81-109. 
P. Chambert-Protat, « Deux témoins d'Ambroise sur le Psaume 118 et leur ancêtre », 2017

DOI 10.1484/M.USML-EB.5.115037 ; halshs-01631460

très similaire à celle dont Florus fit constamment usage pour préparer ses compilations sur l'Apôtre? .

Or cette collection De fide de Florus puise abondamment dans le corpus ambrosien. Elle s'ouvre sur une transcription intégrale des deux premiers livres du De fide ad Gratianum d'Ambroise (= FLOR. LVGD. fid. 1), suivis de son De incarnationis dominicae sacramento (fid. 2). On trouve ensuite une compilation ambrosienne (fid.3), formée de cinquante extraits de l'Expositio in Lucam suivis de deux courts extraits de l'in psalm. 118 serm. :

- Flor. Lvgd. fid. 3,51 (Montpellier H. 308, f. 68v,10-20) :

«ITEM. Deus deus meus respice... nec dabis sanctum tuum uidere corruptionem. »

= AMBR. in psalm. 118 serm. 1,19 (CSEL 62, p. 18,17-19,3 ;

FIRENZE Plut. XIV. 21, f. 7r,16-26 ; PARIS n.a.1. 1437, p. 13,a,2-18)

- Flor. Lvgd. fid. 3,52 (MONTPELlier H. 308, f. 68v,20-69v,20) :

«ITEM. Ego inquit sum minimus apostolorum... In christo utique honoratur et caro. »

= AMBR. in psalm. 118 serm. 20,16-19 (CSEL 62, p. 453,6-455,23;

FIRENZE Plut. XIV. 21, f. 144r,25-145r,4 ; PARIS n.a.l. 1437, p. 366,b,16-368,b,13)

L'in psalm. 118 serm. n'est donc pas une source très importante de FLOR. LVGD. fid., mais il permet à Charlier de signaler que $\stackrel{650}{=}$

le ms.-source dont Florus s'est servi (...) nous a été indirectement conservé : l'ancien ms. de Cluny, Paris B. N., nouv. acquis. lat. 1437, du Xe siècle, en est certainement la copie directe, car

7. Quinze compilations de Florus sur l'Apôtre sont conservées, tirées chaque fois d'un corpus patristique différent. [1] L'Expositio tirée d'Augustin. Grand succès au Moyen Âge central, elle est conservée par quatre-vingt témoins dont une partie de l'original, une copie intégrale par un proche de Florus, et trois autres témoins du IX ${ }^{e}$ siècle. Sa première édition critique est en cours : Flori Lugdunensis Expositio in epistolas beati Pauli ex operibus s. Augustini. Pars III : In epistolam secundam ad Corintbios. In epistolas ad Galatas, Ephesios et Philippenses, éd. par P.-I. Fransen, L. De Coninck, B. Coppieters 't Wallant et R. Demeulenaere, Turnhout, 2011 (Corpus Christianorum Continuatio Mediaeualis, 220B ; Flori Lugdunensis Opera omnia, 6). [2-13] Une collection de douze compilations (dont la compilation tirée d'Ambroise : cf. infra). Très peu diffusée, elle a récemment été éditée pour la première fois : Flori Lugdunensis Collectio ex dictis XII Patrum, 3 t., éd. par P.-I. Fransen et B. Coppieters 't Wallant (t. 1-3) et R. Demeulenaere (t. 2-3), Turnhout, 2002-2007 (CCCM 193, 193A, 193B ; FLOO 1-3). [14-15] Une collection de deux compilations, tirées respectivement de Jérôme et de Grégoire le Grand. Également très peu diffusée, elle est encore inédite. On conserve également les préparatifs d'une seizième compilation, tirée d'Eucher de Lyon, qui n’a peut-être jamais été achevée. 
P. Chambert-Protat, « Deux témoins d'Ambroise sur le Psaume 118 et leur ancêtre », 2017

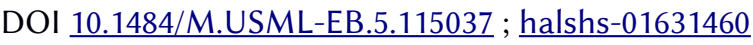

le, ou plutôt les scribes de ce beau volume se sont appliqués avec le plus grand soin à recopier, non seulement les signes et crochets divers de Florus, mais encore ses références ou ses annotations marginales.

Il ajoute alors un seul élément précis, mais assez probant : la section FLOR. LVGD. fid. 3,51 se retrouve, méticuleusement isolée par les crochets habituels, entre les mots deus deus meus, respice... (page 13, col. a, ligne 2) et ...uidere corruptionem (page 13, col. a, ligne 18). En marge, l'indication in euangl. et... les deux initiales, en capitales rustiques, D. F. !...8

PARIS n.a.l. 1437 est donc une copie clunisienne du codex sur lequel Florus avait travaillé. Comme dans d'autres cas similaires, l'antigraphe annoté par Florus est désormais perdu. Après ces conclusions de Charlier, deux études successives permirent de préciser l'environnement clunisien de PARIS n.a.l. 1437.

\section{8 : l'étude paléographique de Garand}

Un quart de siècle s'écoule encore avant que Monique-Cécile Garand ne livre une très importante étude paléographique sur le milieu de production de notre manuscrit clunisien ${ }^{9}$. En comparant les mains des manuscrits à celles des chartes clunisiennes de l'abbatiat de Maïeul dont les scribes se sont nommés, elle parvient à identifier nommément trois des copistes de PARIS n.a.l. 1437 : Theotmar, Ioslenus et Herimannus. D'autres copistes restent anonymes ; mais en recoupant les périodes d'activité des trois qu'elle identifie, elle parvient à la conclusion que notre codex a été copié entre 978 et 994.

\section{3 : Ambroise à Cluny, d'après von Büren}

Enfin au début des années 1990 paraissent les études de Veronika von Büren sur la bibliothèque de Cluny. Elle reconstitue d'abord le catalogue de la seconde moitié du XI ${ }^{e}$ siècle ${ }^{10}$, puis consacre une étude spéciale à la section du catalogue consacrée à Ambroise de Milan ${ }^{11}$. Elle fait observer que le catalogue ne recense pas un seul, mais bien deux exemplaires d'AMBR. in

8. CHARLIER, «Une œuvre inconnue », p. 101-102.

9. M.-C. GARAND, "Copistes de Cluny au temps de saint Maïeul (948-994) », dans Bibliotbèque de l'École des Chartes 136 (1978), p. 7-36.

10. V. VON BÜREN, «Le grand catalogue de la bibliothèque de Cluny », dans Le gouvernement d'Hugues de Semur à Cluny. Actes du colloque scientifique international, Cluny, septembre 1988, publiés par la Ville de Cluny et le Musée Ochier, Mâcon, 1990, p. 245-263 ; EAD., "Le catalogue de la bibliothèque de Cluny du XI siècle reconstitué », dans Scriptorium 46 (1992), p. 256-267. 
P. Chambert-Protat, « Deux témoins d'Ambroise sur le Psaume 118 et leur ancêtre », 2017 DOI 10.1484/M.USML-EB.5.115037 ; halshs-01631460

psalm. 118 serm. Or le mauriste Anselme Le Michel a vu à Cluny, dans les années 1640, un exemplaire de ce texte qu'il dit «Vetustissimum»: un qualificatif qu'il réserve en général, dit Veronika von Büren, aux manuscrits antérieurs à la fondation de Cluny. Ce ne serait donc pas PARIS n.a.l. 1437 qu'il décrirait par là, mais plutôt l'autre exemplaire,

sous lequel on pourrait même soupçonner le manuscrit disparu du diacre Florus, le modèle de BN nal 1437. (...) On peut s'imaginer que les clunisiens ont 'oublié' de rendre le modèle ambrosien emprunté à Lyon, comme cela leur est arrivé dans d'autres cas connus ${ }^{12}$.

Pour von Büren donc, PARIS n.a.l. 1437 a peut-être voisiné plusieurs siècles durant avec son modèle, un exemplaire « vetustissimum » qui n'aurait disparu qu'après la visite de Le Michel.

Ainsi quatre-vingts années après l'édition d'AMBR. in psalm. 118 serm., on a sur le manuscrit de Cluny un luxe d'informations d'une remarquable qualité, qui affinent considérablement la description sommaire de Petschenig. En revanche, personne ne parait avoir réexaminé notre autre témoin, FIRENZE Plut. XIV. 21, puisque la datation erronée de Petschenig en détournait tous ces chercheurs. $\stackrel{\underline{65}}{ }$

\section{8 : le catalogue posthume de Bischoff}

C'est à la paléographie et à l'œil sans égal de Bernhard Bischoff qu'on doit la correction de cette erreur. Mais il n'eut pas l'occasion de l'évoquer de son vivant, et le fait resta insu de tous jusqu'à la parution du premier tome de son catalogue posthume des manuscrits continentaux du $\mathrm{IX}^{\mathrm{e}}$ siècle $^{13}$. Dans la notice qu'il consacre au Plut. XIV. 21, Bischoff qualifie son écriture de «minuscule lyonnaise, époque leidradienne tardive », et par conséquent il lui donne pour origine «Lyon, premier quart du $\mathrm{IX}^{\mathrm{e}}$ siècle ». Mais surtout, il signale « d'innombrables notes marginales de Florus ${ }^{14} »$. À l'évidence, il avait retrouvé l'exemplaire de travail de Florus d'AMBR. in psalm. $118 \mathrm{serm}$.

11. V. VON BÜREN, «Ambroise de Milan dans la Bibliothèque de Cluny », dans Scriptorium 47 (1993), p. 127165.

12. VON BÜREN, «Ambroise de Milan », p. 135.

13. B. BischOFF ( $\dagger$ ), Katalog der festländischen Handschriften des neunten Jabrbunderts (mit Ausnabme der wisigotischen), t. 1 : Aachen-Lambach, Wiesbaden, 1998.

14. «Lyoneser Minuskel, spätere Leidrad-Zeit. (...) Zahlreiche Marginalien von Florus. (...) Lyon, IX. Jh., 1. Viertel » BISCHOFF, Katalog, p. 258, s.n. 1220. 
P. Chambert-Protat, « Deux témoins d'Ambroise sur le Psaume 118 et leur ancêtre », 2017

DOI 10.1484/M.USML-EB.5.115037 ; halshs-01631460

Très belle trouvaille à la vérité, non seulement pour les recherches floriennes, mais aussi pour la tradition d'Ambroise de Milan : le Plut. XIV. 21 devenait subitement le plus ancien témoin complet conservé de l'in psalm. 118 serm. Mais une notice de quatre lignes dans l'immensité d'un tel catalogue est chose discrète et susceptible de passer inaperçue ; d'autant que la datation de Petschenig n'incitait guère les spécialistes d'Ambroise à venir chercher ce témoin dans un catalogue de manuscrits du $\mathrm{IX}^{\mathrm{e}}$ siècle. C'est donc à un spécialiste de Florus, Klaus Zechiel-Eckes, qu'il revint de se saisir du dossier, de mettre en lumière la découverte de Bischoff, et de reprendre à frais nouveaux la question des relations entre FIRENZE Plut. XIV. 21 et PARIS n.a.l. 1437.

\section{9 : l'étude de Zechiel-Eckes}

L'étude de Klaus Zechiel-Eckes est l'une des dernières publications de son vivant. Il disparut en effet prématurément le 23 février 2010, après avoir donné cette contribution au fascicule spécial que la Revue bénédictine consacra à Florus de Lyon au second semestre de $2009^{15}$. Cet article est particulièrement $\stackrel{653}{=}$ important au regard de la présente étude, aussi le lecteur nous excusera-t-il d'en résumer la démarche et les conclusions.

Étant donné ce qu'on savait déjà des deux manuscrits - la parenté textuelle des deux témoins (Petschenig); le fait que PARIS n.a.l. 1437 copie un manuscrit de travail de Florus (Charlier) ; et le fait que FIRENZE Plut. XIV. 21 est un manuscrit de travail de Florus (Bischoff) - il était tout naturel de penser que le Plut. XIV. 21 s'identifiait avec le modèle perdu de PARIS n.a.l. 1437 : c'est l'hypothèse que Klaus Zechiel-Eckes entend tester. L'enjeu n'est pas seulement d'établir les faits. En effet tous les antigraphes floriens de manuscrits clunisiens sont réputés perdus : la découverte de Bischoff semblait donc offrir une occasion unique de comparer original et copie, de surprendre les copistes clunisiens à l'ouvrage, et ainsi de vérifier leur fiabilité.

\section{Bischoff vérifié}

Klaus Zechiel-Eckes montre d'abord que les annotations du manuscrit du Plut. XIV. 21 sont bel et bien de la main de Florus, sans qu'aucun doute soit permis : elles sont nombreuses, et c'est partout sa méthode personnelle, partout sa main caractéristique ; enfin l'on retrouve sans

15. K. ZECHIEL-ECKES, «Eine neue Arbeitshandschrift des Diakons Florus von Lyon. Der Kommentar des Ambrosius zum CXVIII. Psalm (Cod. Firenze, Bibl. Med. Laur., Plut. XIV. 21)», dans Revue bénédictine 119/2 «Florus de Lyon» (2009), p. 336-370. 
P. Chambert-Protat, « Deux témoins d'Ambroise sur le Psaume 118 et leur ancêtre », 2017

DOI 10.1484/M.USML-EB.5.115037; halshs-01631460

peine toutes les minutieuses préparations des extraits d'AMBR. in psalm. $118 \mathrm{serm}$. que Florus a utilisés dans sa compilation ambrosienne sur l'Apôtre (FLOR. LVGD. ex Ambr. in Apost.) :

Es gelingt ohne jegliche Mühe, alle 153 Zitate aus Ambrosius' Psalmenexegese in Cod. Florenz Plut. XIV. 21 nachzuweisen ${ }^{16}$.

Pour aborder la question des rapports entre le Plut. XIV. 21 et PARIS n.a.l. 1437, Klaus Zechiel-Eckes reprend ensuite l'hypothèse de Charlier selon laquelle le manuscrit clunisien serait «certainement la copie directe » du manuscrit de travail de Florus : et il n'a aucune peine à montrer la parenté évidente et indéniable qui existe entre les annotations marginales de l'un et de $\underline{\underline{654}}$ l'autre. PARIS n.a.l. 1437 doit donc bel et bien, conclut-il, descendre du Plut. XIV. 21.

\section{Disparités embarrassantes}

Et pourtant, remarque-t-il, en regard des annotations du Plut. XIV. 21, l'exemplaire de Cluny est une copie très partielle - beaucoup trop partielle. Une écrasante majorité des annotations du Plut. XIV. 21 ne se retrouve aucunement dans PARIS n.a.l. 1437. Cette remarque l'amène d'abord à nuancer la prophétie de Charlier :

Allerdings führte die mehrtätige Autopsie des Parisinus zu gewissen Zweifeln am Sachverhalt einer unmittelbaren Abschrift («certainement la copie directe»). Die Vorbehalte lassen sich zum einen codicologisch begründen, zum anderen aber auch damit rechtfertigen, daß « le plus grand soin », den Charlier beim kopieren der Florus-Adnotationen glaubte beobachten zu können, einer Überprüfung nicht standhält ${ }^{17}$.

Plus gênant encore, il remarque que les copistes clunisiens se sont répartis l'ouvrage d'une façon qui ne saurait correspondre à la composition matérielle du Plut. XIV. 21. Les copistes ont laissé de grands blancs à la fin de certains cahiers, aux endroits où ils étaient convenus de se relayer. En effet, ils avaient distribué entre eux les cahiers déreliés de leur modèle : lorsque l'un

16. ZECHIEL-ECKES, "Eine neue Arbeitshandschrift », p. 344. Ce diagnostic a été nuancé par C. GERZAGUET, «La Collectio ambrosienne de Florus de Lyon : sources d'une compilation et enjeux d'une méthode de travail », dans Mélanges de l'École française de Rome - Moyen Âge 2 (2011), p. 531-543. Mais ces précisions ne sont pas de nature à remettre en cause la conclusion : le Plut. XIV. 21 est bel et bien l'exemplaire d'AMBR. in psalm. 118 serm. sur lequel Florus a préparé les extraits qu'il destinait à son ex Ambr. in Apost. Cette compilation a été éditée par P.-I. FRANSEN, B. Coppieters 't Wallant et R. Demeulenaere (éds.), Flori Lugdunensis Collectio ex dictis XII Patrum. Pars II, Turnhout, 2006 (CCCM 193A).

17. ZECHIEL-ECKES, « Eine neue Arbeitshandschrift », p. 353-354. 
P. Chambert-Protat, « Deux témoins d'Ambroise sur le Psaume 118 et leur ancêtre », 2017

DOI 10.1484/M.USML-EB.5.115037 ; halshs-01631460

avait fini son travail, il n'y avait plus qu'à laisser blanc le reste de la page, puisque la suite du texte avait déjà été copiée sur un nouveau cahier par un de ses confrères. Or dans le Plut. XIV. 21, ces endroits du texte ne correspondent jamais à une frontière de cahier ; bien au contraire ils tombent au beau milieu des pages. En d'autres termes, si les copistes de Cluny s'étaient distribué les cahiers du Plut. XIV. 21, ces passages de relais seraient intervenus en des endroits différents de ceux qu'on observe dans PARIS n.a.l. 1437. $\underline{\underline{655}}$

\section{Décevantes conclusions}

Dès lors pour Klaus Zechiel-Eckes, il est extrêmement peu vraisemblable que Plut. XIV. 21 soit l'ancêtre direct de PARIS n.a.l. 1437, et il est beaucoup plus plausible, sinon nécessaire, de supposer un intermédiaire :

Dies kann zumindest als Indiz dafür gewertet werden, daß die Vorlage des Parisinus n. a. 1. 1437 nicht der Pluteo XIV. 21 war, sondern ein weiteres Zwischenglied einzubauen ist. In genealogischer Betrachtung wäre der Parisinus dann nicht der Sohn, sondern der Enkel des Pluteo $^{18}$.

Compte tenu de l'enjeu annoncé au départ, on comprend la déception dont sa conclusion ne se cache pas : l'existence d'un intermédiaire perdu amoindrit considérablement la valeur du témoin clunisien, à la fois pour le texte d'Ambroise, et pour les études floriennes. Elle ruine en effet toute possibilité d'évaluer la précision avec laquelle les copistes clunisiens copiaient les paratextes floriens, puisqu'il devient impossible de déterminer à quel stade ont été introduites les différences observées entre les deux corpora annotationum ${ }^{19}$.

18. ZECHIEL-ECKES, « Eine neue Arbeitshandschrift », p. 354.

19. «Schließlich war zu zeigen, daß Cod. Paris, Bibliothèque nationale de France, nouv. acq. lat. 1437 (spätes 10. Jahrhundert, Cluny) ohne Zweifel auf den Pluteo XIV. 21 zurückgeht. Allerdings dürfte sich die von Célestin Charlier postulierte direkte Abhängigkeit des Parisinus vom «manuscrit personnel» in Firenze (...) nicht bestätigen : Zwischen Pluteo und Parisinus (also ungefähr zwischen den Jahren 810 und 990) wird man mit großer Wahrscheinlichkeit eine weitere Überlieferungsstufe ansetzen müssen. In der Konsequenz ließ sich ein ursprüngliches Ziel vorliegender Studie, nämlich Schreiber des Klosters Cluny bei ihrer täglichen Arbeit zu beobachten, nicht realisieren. Denn es bleibt ungewiß, welche Veränderungen am Text des Pluteo (aber auch Modifizierungen an den Glossen und Exzerpiervermerken des Diakons Florus) bereits von der hier postulierten, intermediären Stufe zwischen Pluteo und Parisinus zu verantworten sind. » ZECHIEL-ECKES, «Eine neue Arbeitshandschrift », p. 356-357. 
P. Chambert-Protat, « Deux témoins d'Ambroise sur le Psaume 118 et leur ancêtre », 2017

DOI 10.1484/M.USML-EB.5.115037 ; halshs-01631460

\section{Reprendre le dossier}

L'étude de Klaus Zechiel-Eckes est récente, mais dans cet intervalle, les campagnes de numérisation ont mis à la disposition des chercheurs des instruments nouveaux : des reproductions intégrales, en couleur, en haute définition, accessibles gratuitement et simultanément, à volonté, à toute heure du jour ou de la nuit. On ne saurait trop se féliciter de ce

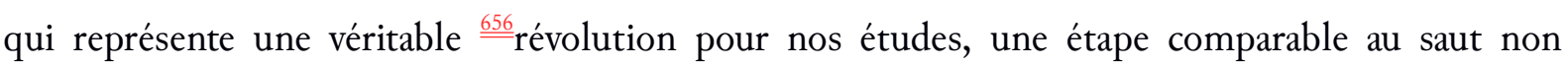
seulement quantitatif mais bel et bien qualitatif que la photographie et le microfilm ont représenté en leur temps.

En l'espèce, nos deux manuscrits sont désormais consultables, chacun sur le site de sa bibliothèque de conservation : il est donc facile de les consulter ensemble et de les comparer feuillet par feuillet. On peut parcourir la reproduction du manuscrit de Paris, alors qu'on étudie le manuscrit de Florence dans la salle de lecture de la Biblioteca Medicea Laurenziana ${ }^{20}$; ou bien la reproduction du manuscrit de Florence tout en se penchant sur le manuscrit de Paris, dans la salle de lecture de la Bibliothèque nationale de France. Autant d'avantages inappréciables dont Zechiel-Eckes n'a pu bénéficier, puisque le Pluteus n'est paru en ligne que le 19 novembre 2009, alors que sa propre étude était sous presse ; et le manuscrit de Cluny seulement après sa mort, le 28 août 2013, dans le cadre de la numérisation générale des manuscrits floriens de la BNF financée par Biblissima.

Or de telles conditions de travail étaient indispensables pour démêler l'écheveau et reconstituer la trame véritable des faits. La couleur en particulier, avantage inappréciable de ces numérisations sur les anciens microfilms, rend manifeste les contrastes et les ressemblances des encres ; et l'on confirme ainsi facilement ce que la seule différence des mains ne rendait sans doute pas assez clair. Un travail de comparaison en vis-à-vis permet ainsi de mettre au jour plusieurs faits et corrélations, qui obligent à réévaluer les conclusions précédentes.

\section{Notes de Florus}

Sans refaire tout le développement par lequel Zechiel-Eckes a vérifié le diagnostic de Bischoff, il faut d'abord redire que l'essentiel du corpus annotationum du Plut. XIV. 21 est non seulement de type florien, mais de la main même de Florus de Lyon en personne.

20. Chose qu'une bourse de l'École française de Rome m’a donné la chance de faire au mois de mai 2014. 
P. Chambert-Protat, « Deux témoins d'Ambroise sur le Psaume 118 et leur ancêtre », 2017

DOI 10.1484/M.USML-EB.5.115037 ; halshs-01631460

On a plusieurs fois souligné le caractère particulier, " reconnaissable entre mille », de cette main. Ce caractère ressort d'abord de la forme des lettres : d'une précision géométrique, elles restent harmonieusement souples ; leurs pleins sont aussi renflés que leurs déliés sont fins ; elles paraissent à la fois fermes et nerveuses, comme pleines d'une énergie contenue. Mais on la recon ${ }^{\frac{657}{5}}$ naît aussi à un certain nombre de « tics » graphiques, qui n'ont rien de nécessaire et dont Florus se plaît à orner ses notes : points suspendus qui tournent souvent à la virgule, tildes prenant la forme d'M obliques, ou encore l'accolade florienne, ce

signe florien par excellence : un signe fait d'un point entre deux courbes [qui] précède gloses ou références dans les marges. Ce signe est extrêmement répandu dans tous les manuscrits annotés par Florus et signale son intervention : c'est de sa part une véritable signature ${ }^{21}$.

Enfin Florus a une habitude de lecteur qui, alors qu'on pourrait la croire répandue, s'avère tout à fait personnelle ${ }^{22}$ : l'habitude de marquer dans la marge, sous forme d'un sigle en semionciales, les livres bibliques cités dans le texte qu'il lit.

Main, tics ornementaux, habitudes personnelles de Florus : dans le corpus annotationum du Plut. XIV. 21, plusieurs centaines de notes vérifient ces critères. En particulier, de tous les manuscrits annotés par Florus - originaux ou copies —, c'est celui où l'on observe la plus grande diversité de livres bibliques siglés en marge. Ces notes sont portées dans une encre très foncée, presque noire, qui contraste très fortement avec l'encre notablement pâle du copiste. C'est la même encre qui a aménagé dans le texte, en regard des sigles marginaux des épîtres pauliniennes, les dispositifs d'excerption préparant les cent quarante-quatre extraits rubriqués «Ex tractatu psalmi 118 » de la compilation ambrosienne de Florus sur l'Apôtre ${ }^{23}$ : crochets $^{2}$ découpant le texte (paragraphus et positura, selon la terminologie d'Isidore de Séville ${ }^{24}$ ), avec un

21. L. HoLTZ, "La minuscule marginale et interlinéaire de Florus de Lyon», dans Gli autografi medievali. Problemi paleografici e filologici, Atti del convegno di studio della Fondazione Ezio Franceschini, Erice, 25 settembre - 2 ottobre 1990, Spoleto, 1994 (Quaderni di cultura mediolatina, 5), pp. 149-166, avec huit planches ; ici p. 153 ; des exemples sont reproduits dans les figs. 1-23.

22. Peut-être quelques émules de son environnement immédiat ont-ils pratiqué cet exercice de façon sporadique ; mais personne ne l'a fait avec la constance de Florus.

23. Le nombre de 153 donné par Klaus Zechiel-Eckes est faux. L'ex Ambr. in Apost. donne 150 extraits dont le texte remonte à l'in psalm. 118 serm., mais six d'entre eux sont placés sous une rubrique «In expositione Cantici canticorum », et le Plut. XIV. 21 ne porte pas leurs préparations. J'expliquerai ailleurs l'origine de ces six extraits. 
P. Chambert-Protat, « Deux témoins d'Ambroise sur le Psaume 118 et leur ancêtre », 2017

DOI 10.1484/M.USML-EB.5.115037; halshs-01631460

recours systématique au système de «points de suspension» élaboré par Florus $\stackrel{658}{=}$ lui-même ${ }^{25}$; ponctuation détaillée; corrections orthographiques, voire textuelles lorsque cela s'impose. Le contraste entre cette encre et celle du copiste est tel, qu'en ouvrant le volume au hasard on voit immédiatement les pattes de mouches de Florus ressortir sur la page.

\section{Un exemple}

Je ne prendrai qu'un exemple, celui du f. 108r. Les cinq premières lignes du texte sont vierges de toutes traces de l'encre noire : Florus n’y a pas touché. En revanche à partir de la ligne 7 , cette encre noire révise systématiquement toute la ponctuation du texte : certains signes sont précisés (le punctus de la subdistinctio est changé en punctus eleuatus pour la media distinctio ou en punctus flexus pour la distinctio), d'autres signes sont corrigés (punctus flexus devenu punctus eleuatus, ou inversement; signes d'interrogation barrés de deux petits traits obliques, 1.13 et 1. 28), d'autres signes encore sont tout bonnement ajoutés, insérés de force entre les mots du copiste ; certains signes enfin sont tout bonnement annulés, au moyen d'un trait horizontal qui, tiré sur la ligne inférieure de l'écriture, paraît vouloir rétablir une sorte de scriptio continua (1. 11, 1. 12, 1. 17).

Tout en révisant méticuleusement la ponctuation, cette encre noire revoit aussi l'orthographe du passage (les «heredis» et «hereditas» du copiste sont systématiquement corrigés en «hęred-», seize fois sur les 1. 14-15, 18, 20-21, 23, 25-28, 31 ; «oberratos » en «obęratos », 1. 16 ; « conditio » en " condicio », 1.24), mais aussi sa grammaire (« terrestris » du copiste est corrigé en «terrestri », 1. 10 ; « his qui » en « is qui », 1. 10), et son texte lui-même ( "hereditas est damnum discriptio coheredis » du copiste est corrigé en « hęredis est damnum. adscriptio cohęredis », 1. 25-26). Enfin là où il est écrit "mala euae », la même encre noire a rajouté entre les deux mots un trait oblique discontinu ("malaieuae », 1. 17) qui, dans le système de correction de Florus, sert à éviter les mécoupures. Le risque n’était pourtant pas élevé ici, puisque «malæ væ» ne ferait pas grand sens et ne parait pas devoir s'imposer spontanément à l'esprit d'un copiste, aussi distrait soit-il — et d'ailleurs le copiste du Plut. XIV. 21 avait $\stackrel{659}{=}$ bien

24. ISID. etym. 1,21 : W.M. LINDSAY (éd.), Isidori Hispalensis Etymologiarum sive Originum libri XX, 2 t., Oxford, 1911 (Oxford Classical Texts), t. 1, p. 48-49.

25. Selon le schéma : [incipit... suspension]“ passage non retenu “[reprise... suspension]" passage non retenu ..[reprise... explicit]. Le procédé a été mis au jour par CHARLIER, «Les manuscrits personnels ». 
P. Chambert-Protat, « Deux témoins d'Ambroise sur le Psaume 118 et leur ancêtre », 2017

DOI 10.1484/M.USML-EB.5.115037 ; halshs-01631460

laissé un petit intervalle au bon endroit entre les deux mots. Aussi y a-t-il dans cette «correction» préventive un scrupule véritablement tâtillon ${ }^{26}$.

Pourquoi donc une telle attention portée au texte, et pourquoi seulement à partir de la septième ligne de ce feuillet ? C'est qu'à la sixième ligne on trouve, de la même encre noire, une paragraphus qui marque le début d'un passage à extraire. Une positura clôt ce passage à la treizième ligne, mais dès la ligne suivante une nouvelle paragraphus signale le début d'un nouveau passage à extraire, qui se prolonge sur tout le bas de la page et jusqu'au verso (sa positura se situe f. 108v,9). Entre la positura du premier passage (1.13) et la paragraphus du second (1.14), un membre de phrase est donc resté exclu des deux extraits : membre de phrase où la ponctuation n'a pas été révisée, et où le mot « hereditate » n'a pas reçu sa cédille à l'encre noire. La révision de la ponctuation et de l'orthographe est donc bien liée, directement et exclusivement, à la préparation des deux extraits balisés par des paragraphi et positurae. Un autre raffinement s'observe dans le découpage du second extrait. Quelques mots après la grande paragraphus qui marque le début du passage à extraire, l'excerpteur a tracé une positura de module très inférieur, et presque aussitôt suivie d'une paragraphus symétrique : il a ainsi retranché un seul mot, «ita » (1. 14), qui rattachait le passage à son contexte dans le traité d'Ambroise, et qui était voué à ne plus rien signifier quand il en serait extrait ${ }^{27}$.

Enfin la même encre noire a laissé deux annotations dans la marge du f. 108r. À la hauteur de la phrase d'Ambroise: [deus] «apud quem et conscientia tua testis est' cogitationibus accusantibus aut etiam defendentibus.' ( $1.7-8)$ située au début du premier extrait, la marge porte un sigle ROM, suivi d'un point, sommé d'un tilde en forme de M oblique, et précédé d'une accolade florienne. L'annotateur a reconnu dans le texte d'Ambroise une citation de Rom. 2,15 : « testimonium reddente illis conscientia ipsorum, et inter se inuicem cogitationibus accusantibus, aut etiam defendentibus. » Or l'extrait préparé ici $\stackrel{660}{=}$ par notre encre noire, avec la minutie qu'on a

26. Pour être tout à fait complet, signalons que Florus a également rajouté un accent sur « foénore » (1. 16), peut-être pour marqué la diérèse (comme en français le tréma de Noël, Raphäl, etc.). L'usage de l'accent chez Florus n’a pas encore été analysé ni même, je crois, signalé. Il l'utilise sur les transcriptions latines de mots grecs, mais en timbre aussi très souvent les monosyllabes latins, comme pour les renforcer (par exemple « ví »). Il semble aussi que parfois l'accent lui serve à éviter d'éventuelles mécoupures, lorsqu'il suffit à supprimer l'ambiguïté.

27. On pourrait résumer ces découpages ainsi : [ideoneus ... factum est] et bene ... sicut [ante ... peccatoris ${ }^{\text {ita }}$ [nunc ... reformatio]. En l'occurrence, Florus n'a pas eu besoin de recourir au système des « points de suspension » (cf. n. 23). 
P. Chambert-Protat, « Deux témoins d'Ambroise sur le Psaume 118 et leur ancêtre », 2017

DOI 10.1484/M.USML-EB.5.115037 ; halshs-01631460

vue, commente précisément ce verset dans FloR. LvGD. ex Ambr. in Apost. exc. 12 in Rom. XII ${ }^{28}$.

La seconde annotation marginale conduit aux mêmes conclusions. À la hauteur de cette phrase : " (sicut) ante- hęredes fuimus peccatoris.' (ita) nunc hęredes sumus christi·, » (1. 14) qui forme l'incipit du second extrait (sauf les mots entre parenthèses, que l'excerpteur a écartés), la marge porte un nouveau sigle ROM tout à fait similaire au premier. Florus a vu là une allusion à Rom. 8,17, "si autem filii, et haeredes : haeredes quidem Dei, cohaeredes autem Christi » : et c'est ce verset que commente ce second extrait, dans l'ex Ambr. in Apost., exc. 37 in Rom. XXXVII ${ }^{29}$. Ainsi l'exemple du seul f. $108 \mathrm{r}$ suffit amplement à démontrer que Florus en personne a travaillé sur le codex FIRENZE Plut. XIV. 21.

\section{Notes « floriennes »d'une autre main}

Tout le travail autographe de Florus est si présent et si reconnaissable dans ce codex, que par contraste un certain nombre d'autres notes laissent d'autant plus perplexes. Il ne s'agit évidemment pas des traces laissées çà et là par quelques lecteurs beaucoup plus récents, mais d'un corpus annotationum qui parcourt l'ensemble du codex et qui s'y trouvait avant même que Florus l'annote ; qui n'est pas de la main de Florus et qui, pourtant, présente toutes les caractéristiques du travail de Florus, à telle enseigne que Klaus Zechiel-Eckes l'a confondu avec le corpus autographe de Florus.

Tous ces caractères se reconnaissent en particulier à l'examen des sigles scripturaires. À titre d'exemple, j'ai compté sur l'ensemble du manuscrit, toutes mains confondues, soixante-trois sigles des épîtres aux Corinthiens ${ }^{30}$. Sur l'ensemble, quarante-sept (près de $75 \%$ ) sont attribuables à Florus lui- $-\frac{661}{m}$ même. Ils se présentent tous sous la forme COR $\cdot \mathrm{I} \cdot$ ou $\mathrm{COR} \cdot \mathrm{II} \cdot-$ celle

28. CCCM 193A : le verset commenté se lit dans la table des capitula de l'épître aux Romains, p. 3, s.n. XII ; l'extrait lui-même se lit p. 12.

29. Ibid., p. 4 s.n. XXXVII pour le verset commenté, p. 28-29 pour l'extrait proprement dit.

30. Les sigles des épîtres aux Corinthiens constituent le meilleur échantillon-test, puisqu'il s'agit d'un objet paléographique d'une teneur dirimante (contrairement aux Nota), dont la forme est remarquablement stable (contrairement aux notes), et dont les apparitions sont les plus récurrentes. En effet, dans toutes les compilations de Florus sur l'Apôtre sauf les plus chétives, les deux épîtres aux Corinthiens rassemblent beaucoup plus d'extraits patristiques que les autres épîtres : cela suppose que Florus a préparé des extraits pour ces épîtres plus souvent que pour aucune autre, et donc porté ce sigle dans les marges de ses manuscrits plus souvent qu'aucun autre. 
P. Chambert-Protat, « Deux témoins d'Ambroise sur le Psaume 118 et leur ancêtre », 2017 DOI $\underline{10.1484 / M . U S M L-E B .5 .115037}$; halshs-01631460

que Florus leur a donnée dans les autres manuscrits-sources de ses compilations sur l'Apôtre. Ils sont presque tous sommés de son tilde en forme d'M oblique, et presque tous précédés de l'accolade florienne ; ils sont tracés dans cette encre très foncée qu'on a remarquée ; leur main est indéniablement celle de Florus ; et chacun d'eux correspond à la préparation d'un extrait qu'on retrouve dans l'ex Ambr. in Apost., in I Cor. ou in II Cor.

Le quart restant - seize sigles — est plus disparate. Sur tout le début du manuscrit, ils portent la préposition «ad»: «adcor· » (sept fois), «adcorintios » (une fois), toujours avec A et D onciaux, mais avec 2 ( $r$ rotunda) final en ligature. Le dernier de la sorte se situe f. $57 \mathrm{r}$. L'occurrence suivante, f. 78r, n'a plus la préposition, et les sept suivants non plus ; en revanche l'r final en ligature tend à disparaittre au profit d'un petit $\mathrm{R}$ oncial.

Formellement, ils sont très proches des sigles autographes de Florus ; mais ce n'est pas le seul détail qui fasse penser à lui. Trois de ces sigles (tous avec «ad») sont accompagnés d'un motif en zigzag, qui évoque beaucoup les encadrements ondulés que Florus donne à ses annotations lorsqu'elles s'approchent trop près du corps du texte. Quatre autres sigles (un avec «ad», trois sans) sont couverts d'une ample potence, qu'on observe aussi parfois sur les sigles scripturaires autographes de Florus, en lieu et place de l'accolade florienne (qui elle-même n'est pas systématique). Chaque sigle a un petit signe de conclusion (généralement un punctus, deux fois un punctus flexus, une fois une sorte de M malhabile renversé d'un quart de tour dans le sens dextre) ; tous sont sommés d'un tilde allongé (sauf la mention «adcorintios », puisque ce n'est pas une abréviation, et un sigle COR où l'absence du tilde peut s'expliquer par une simple omission), avec des ondulations parfois très marquées.

Pourtant, ils ne sont pas de la main de Florus. La forme des caractères est très différente. L'o est plus menu, presque mangé par le $c$ ou par l'r, là où celui de Florus se détache toujours nettement. De manière générale, dès que cette main s'essaie aux caractères de forme onciale, elle devient beaucoup moins régulière : il n'en va pas de même chez Florus, qui mélange naturellement les formes, sans peine et sans rien perdre de sa constance. Enfin, ces seize sigles ne sont pas tracés dans l'encre si foncée dont on a parlé ; ils sont au contraire d'une encre très pâle, très proche de celle du copiste.

On s'en aperçoit d'autant plus facilement que les autres annotations du codex juxtaposent souvent, elles aussi, les deux encres : l'encre noire réexamine les notes à l'encre pâle, les amende, les précise, les complète. Inversement, les $\frac{662}{n}$ notes à l'encre foncée ne sont jamais révisées par 
P. Chambert-Protat, « Deux témoins d'Ambroise sur le Psaume 118 et leur ancêtre », 2017

DOI 10.1484/M.USML-EB.5.115037 ; halshs-01631460

l'encre pâle. Nos seize sigles des Corinthiens permettent eux-mêmes de s'en faire une idée : onze d'entre eux sont retouchés à l'encre foncée. Le réviseur ajoute le numéral $\cdot \mathrm{I} \cdot \mathrm{ou} \cdot \mathrm{II} \cdot$ qui manquait (quatre fois), ou corrige le $\cdot \mathrm{I} \cdot$ en $\cdot \mathrm{II} \cdot$ (une fois) ; il ajoute sur la ligne l'b oubliée dans le sigle "adcorintios ». Une fois, il barre même franchement le sigle (un long trait horizontal et deux traits obliques). Parfois, il se contente d'ajouter tel ou tel détail purement ornemental : il ajoute une virgule flottante pour transformer un $\mathrm{COR} \cdot \mathrm{II} \cdot$ en $\mathrm{COR} \cdot \mathrm{II} \cdot$ ' ou bien, lorsque le sigle n'est pas précédé d'une potence ou d'un zigzag, il lui confère l'accolade florienne.

Seul Florus pouvait porter ce genre d'attention à ces sigles scripturaires : bon nombre de ces révisions s'efforcent en fait de conformer les sigles de l'autre main aux usages personnels de Florus. On a observé en outre que chacun des quarante-sept sigles autographes de Florus a fourni un extrait à l'ex Ambr. in Apost. : or parmi les seize sigles non autographes, chacun des onze sigles retouchés par Florus accompagne la préparation d'un extrait sur l'Apôtre ; tandis que trois des cinq sigles non retouchés n’ont pas non plus servi à de telles préparations. La révision des sigles, leur conformation aux usages personnels de Florus, est donc largement corrélée à la préparation minutieuse, sur le codex FIRENZE Plut. XIV. 21 et par Florus lui-même, des extraits d'AMBR. in psalm. 118 serm. que Florus destinait à sa compilation ex Ambr. in Apost.

Ce diagnostic posé sur les seuls sigles aux Corinthiens peut être étendu à l'ensemble du corpus annotationum florien du Plut. XIV. 21 : non seulement les autres sigles pauliniens, mais aussi les autres sigles scripturaires et même les notes plus développées. Le codex en présente beaucoup plus de la main même de Florus que de l'autre main, mais les notes de cette dernière demeurent assez nombreuses pour qu'on les étudie. Ses Nota ont généralement la même forme que ceux de Florus - on peut les comparer aux Nota autographes de Florus du même codex : une $\mathrm{N}$ capitale trapue dont la seconde haste verticale, très haute, porte l'horizontale amplement ondulée du T ; aucune des deux voyelles, mais toujours un ornement : un punctus, une ou deux apostrophes, ou bien une virgule très ronde ou l'M oblique de Florus, flottant sur le tout.

Les encadrements ondulés reviennent eux aussi de loin en loin, dans les notes de l'autre main comme dans celles de la main de Florus. On peut comparer en particulier la grande note du f. $18 \mathrm{v}$, « Nota diligenter de igne purgatorio et leuioribus admissis », avec celle du f. 145r, "Istam tu sancte pater eloquisti. $\stackrel{\underline{663}}{\mathrm{Ut}}$ diceres moriens: nec timeo mori, quia bonum dominum 
P. Chambert-Protat, « Deux témoins d'Ambroise sur le Psaume 118 et leur ancêtre », 2017 DOI $\underline{10.1484 / M . U S M L-E B .5 .115037}$; halshs-01631460

habemus ${ }^{31} »$. La seconde est de la main de Florus : elle s'ouvre sur une accolade florienne, descend en une colonne étroite (neuf lignes de sept ou huit caractères) tout en s'écartant graduellement du corps du texte, peut-être dans le but d'éviter le sigle scripturaire PTR. (" epistula Petri») qui se trouvait là ; et elle se retranche derrière un long trait ondulé. La première a une forme très similaire : une étroite colonne (neuf lignes de cinq à six caractères) dans un encadrement de deux longs traits ondulés verticaux, refermé au sommet par une barre horizontale, et au pied par la réunion en $V$ des deux verticales - mais elle n'est pas du tout de la main de Florus.

La plupart des notes de "l'autre main » sont intactes, mais toutes n'ont pas échappé à la révision de la main de Florus - et comme pour les sigles, l'inverse ne se produit jamais. Dès le f. 2r, quelques lignes sous une note à l'encre pâle qui n'est manifestement pas de la main de Florus («Nota de oblatione primogenitorum»), la même encre pâle avait porté un Nota isolé : Florus l'a prolongé en ces termes, « de tempore offerendi ab his qui nuper sunt baptizati ». C'est de cette même façon qu'en sept autres endroits Florus complète les Nota de l'autre main, comme pour les gloser, en précisant pourquoi l'endroit est digne d'intérêt.

1. Au f. 19v, Nota de l'autre main, «contra arrianos » de la main de Florus.

2. Au f. 28r, Nota de l'autre main, « de origenis peritia » de la main de Florus.

3. Au f. 28v, Nota de l'autre main, « de malo loquacitatis» de la main de Florus.

4. Au f. 62r, Nota de l'autre main, « de impiis uenatorum » de la main de Florus.

5. Au f. $74 \mathrm{v}$, Nota de l'autre main, « res multum miras » de la main de Florus.

6. Au f. $88 \mathrm{v}$, Nota de l'autre main, « de sphera cęli, et meatu solis » de la main de Florus.

7. Au f. $149 \mathrm{r}$, Nota de l'autre main, « de pactis mime rescindendis » de la main de Florus.

Rarement, la part de l'autre main est un peu plus importante : au f. $57 \mathrm{v}$, « Nota multum » de l'autre main, «de iniqua misericordia » de celle de Florus. Au f. 17v, l'autre main avait écrit « Nota rem miram », avec un encadrement; et dans l'espace qui restait à droite avant le corps du texte, Florus a complété : «de igne futuri iudicii »- non sans compléter l'encadrement, d'un long trait ondulé.

31. PaVl. Med. vita Ambr. 45, 2 (Vita di Cipriano. Vita di Ambrogio. Vita di Agostino. Introduzione di Ch. Mohrmann, testo critico e commenta a cura di A.A.R. Bastiaensen, traduzioni di L. Canali e C. Carena [Milano, 1997 : Vite dei Santi, 3], p. 112, 1. 10-12. 
P. Chambert-Protat, « Deux témoins d'Ambroise sur le Psaume 118 et leur ancêtre », 2017

DOI 10.1484/M.USML-EB.5.115037 ; halshs-01631460

\section{Premier bilan}

Avant que Florus n'ajoute de sa main quantité de notes (cinq cent quarante à cinq cent cinquante, selon mon décompte), le codex portait donc déjà un corpus annotationum assez remarquable : quelque deux cent soixante-quinze à deux cent quatre-vingts notes appartiennent à « l'autre main », celle qui annotait à l'encre pâle. Bien plus, Florus s'est intéressé de très près à ce corpus annotationum, ne se contentant pas de le préciser, de le gloser ou de l'amplifier, mais allant jusqu'à corriger voire biffer franchement certaines notes. Enfin il l'a pleinement intégré à son propre travail de compilateur, puisqu'une partie des activités du premier annotateur forme comme les prémices des travaux que Florus a menés à bien en personne sur ce codex.

Or, si le premier phénomène se rencontre en d'autres occurrences, le second est tout à fait inusité. Comme Florus annotait des manuscrits parfois très anciens, la probabilité qu'ils aient déjà été annotés auparavant par d'autres lecteurs est assez élevée. Dans le codex LYON, BM, 610 par exemple, un exemplaire du Contra Faustum d'Augustin, il est très net que Florus passe derrière un précédent annotateur dont la main, quoique très différente, ne doit pas être beaucoup plus ancienne que la sienne. Là aussi, Florus remploie certains Nota de son prédécesseur pour les prolonger d'une glose. Mais il n'altère pas autrement ce premier corpus de notes : il ne le corrige pas, ne le modifie pas, ne biffe rien. En particulier, le premier annotateur ne s'était pas avisé de sigler dans les marges les livres bibliques, pas même les seules épîtres pauliniennes. Florus n'a donc pas matière à remployer son travail dans sa propre quête de passages sur l'Apôtre : il se contente de rajouter ses propres sigles et ses propres notes, par centaines, amplifiant ainsi démesurément le corpus annotationum du codex.

Le caractère propre de FIRENZE Plut. XIV. 21 tient donc à la manière dont Florus a tenu compte du codex qu'il avait entre les mains, annotations comprises. Et cette manière singulière est directement liée à l'origine du premier corpus de notes, le corpus de « l'autre main ».

\section{Origine du premier corpus de notes - et du codex lui-même}

On a déjà vu que par de nombreux détails graphiques, les notes de cette « autre main »

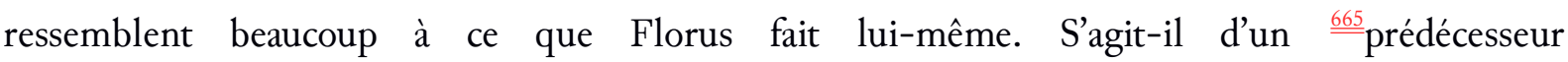
particulièrement proche ? En réalité, d'autres détails nombreux permettent d'établir que ces notes ne sont pas le fait d'un annotateur à proprement parler - quelqu'un qui lit un livre pré-existant et ajoute du texte dans ses marges - mais bien du copiste du codex. 
P. Chambert-Protat, « Deux témoins d'Ambroise sur le Psaume 118 et leur ancêtre », 2017

DOI 10.1484/M.USML-EB.5.115037 ; halshs-01631460

\section{Des notes copiées par le copiste}

Le premier détail est encore la couleur de l'encre, dont il a été question : alors que celle de Florus est très noire et dénote sur la page, l'encre de ces annotations est d'une pâleur et d'une teinte systématiquement identiques à celles du corps du texte. La main aussi est celle du copiste : on peut bien en juger dans certaines notes de la même encre plus développées que les sigles scripturaires. Au f. 2r, 4, les mots "primogenita» sur la ligne et «primogenitorum » dans la marge ont manifestement été écrits par la même personne (à la ligne 10 en revanche, le mot «babtizatus» dans le texte est bien différent du «baptizati » que Florus a porté dans une note marginale autographe). Au f. 2v, 30, le «cantico» de la marge est de la même main que le « canticis » du texte. Au f. 91r, 13-14, le mot « esaie » est identique dans le corps du texte et dans l'annotation marginale à l'encre pâle. Même remarque au f. 115r, 2 pour le mot «apostata », au f. $116 r, 6$ pour « edurus ».

En outre le manuscrit que notre copiste copiait si minutieusement, notes comprises, n'était pas seulement annoté : il portait déjà, lui aussi, des paragraphus et positurae délimitant certains passages du texte. Là encore, on distingue assez nettement celles que Florus a ajoutées de sa main, postérieurement à la copie, et un certain nombre de crochets que le copiste a copiés luimême d'après son modèle. La couleur des encres aide à faire cette distinction, mais ce n'est pas le seul indice. Le travail d'extraction autographe de Florus est manifestement ajouté au codex déjà achevé : comme le copiste ne savait pas où l'on voudrait, plus tard, insérer des crochets d'extraction, Florus a souvent été obligé de «forcer le passage » entre les mots, lettres et signes du texte. Inversement, les crochets du copiste sont souvent installés commodément dans la ligne de texte, copiés pour ainsi dire comme un caractère pareil aux autres. Dans plusieurs cas, on voit même que la personne qui a tracé ces signes n'en comprenait pas le sens. En plusieurs endroits, une paragraphus est ainsi isolée en fin de ligne, alors que le mot qu'elle marque est rejeté par la justification au début de la ligne suivante. Ainsi au f. $3 \mathrm{v}$, la ligne 11 s'achève sur «mystica ${ }^{666}$ dicens. [" tandis que le premier mot de l'extrait, «introduxit », est au début de la ligne 12. Au f. 10v, la ligne 17 s'achève sur «Et alibi [", et le premier mot du passage, "pie », est au début de la ligne suivante. Dans ces cas par excellence le crochet est traité comme un caractère qui se suffirait à lui-même, ce qui contredit directement sa fonction : en tant que signes d'extraction, ces crochets n'ont pas de valeur par eux-mêmes, mais ils confêrent une certaine valeur au mot qu'ils marquent : «l'extrait commencera avec ce mot»; «avec ce mot finira l'extrait». Dans les 
P. Chambert-Protat, « Deux témoins d'Ambroise sur le Psaume 118 et leur ancêtre », 2017

DOI 10.1484/M.USML-EB.5.115037; halshs-01631460

cas donnés en exemple, un lecteur qui aurait voulu ajouter une paragraphus dans le texte d'Ambroise, pour quelque raison que ce soit, l'aurait nécessairement portée au début de la ligne suivante, sur le mot qui l'intéressait. La personne qui a tracé ces crochets n'est donc pas un lecteur, quel qu'il soit, mais bien le copiste du codex, qui recopiait sans bien les comprendre des crochets déjà disséminés sur son modèle ${ }^{32}$.

Finalement les notes, sigles et signes copiés par le copiste de notre Plut. XIV. 21, si «floriens» dans leur conception et si peu «floriens»dans leur exécution, correspondent exactement à ce qui permet habituellement d'identifier un manuscrit copié sur un exemplaire de travail de Florus. Avant de devenir l'exemplaire de travail de Florus d'AMBR. in psalm. 118 serm., le codex FIREnZE Plut. XIV. 21 était donc, en réalité, la copie d'un précédent exemplaire de travail de Florus d'AMBR. in psalm. 118 serm.

\section{Les deux exemplaires de Florus et celui de Cluny}

L'existence successive de ces deux exemplaires jette une lumière nouvelle sur PARIS n.a.l. 1437, lui aussi copie d'un exemplaire de travail de Florus d'AMBR. in psalm. 118 serm. Il n'est pas difficile de confirmer ce que l'on pressent désormais : en effet les notes copiées par les copistes de PARIS n.a.l. 1437 correspondent presque systématiquement à des notes copiées par le copiste du Plut. XIV. 21 ; tandis que les notes que Florus a rajoutées de sa main sur le Plut. XIV. 21 ne trouvent presque jamais de correspondance dans le corpus de notes du PARIS n.a.l. 1437.

${ }^{667}$ Il ne saurait être question d'analyser ici le fait en détail : c'est au total un millier de notes, sans parler des signes et symboles parfois mystérieux, qu'il s'agit de collationner. La coïncidence massive qu'on a décrite rapidement souffre en effet quelques exceptions, qui s'expliquent souvent par des omissions ou des méprises de la part des copistes de l'un et l'autre codex : on a déjà fait remarquer qu'ils ne comprenaient pas toujours très bien ce qu'ils copiaient. Ainsi en rétablissant ici ou là une note que le copiste du Plut. XIV. 21 avait omise ou mal placée, Florus a parfois porté une note qui correspond exactement, de facto, à une note du PARIS n.a.l. 1437 : mais ces très rares circonstances, explicables par d'autres biais, ne pèsent guère en regard des centaines

32. Fait significatif, d'ailleurs, les copistes clunisiens du PARIS n.a.l. 1437 ont commis la même erreur en plusieurs endroits : p. 19, col. a, 1. 19, «quasi pius [» puis 1.20 « responde»; p. 95, b, 8 «tenebant [ " puis 1.9 «Venit»; p. 353, a, 16 « exagitet. [» puis 1.17 «In cubili ». Sur l'ensemble du codex, j'en ai compté une dizaine. Les copistes clunisiens eux aussi copiaient un manuscrit annoté avec son corpus de notes ; et eux non plus ne comprenaient pas bien le sens des étranges crochets disséminés dans le texte. 
P. Chambert-Protat, « Deux témoins d'Ambroise sur le Psaume 118 et leur ancêtre », 2017

DOI 10.1484/M.USML-EB.5.115037 ; halshs-01631460

d'autres cas qu'explique la règle générale. Je compte donner ailleurs une analyse plus complète et plus détaillée du phénomène, dont on peut déduire une foule de renseignements sur la physionomie du «premier exemplaire » annoté par Florus.

Mais pour achever de démontrer l'existence de ce premier exemplaire, j'emprunterai ici une autre voie, plus familière aux philologues : les leçons variantes. J'ai déjà dit plus haut que le Plut. XIV. 21 porte les préparations, de la main de Florus, de chacun des 144 extraits rubriqués «Ex tractatu psalmi CXVIII » de sa compilation ambrosienne sur l'Apôtre, FLOR. LVGD. ex Ambr. in Apost. L'exemple du f. 108r a aussi montré que ces préparations ne consistent pas seulement dans le découpage des extraits, mais aussi dans la correction minutieuse de leur orthographe et de leur ponctuation, voire de leur texte même, directement sur l'exemplaire de travail. Or si les leçons du Plut. XIV. 21 post correctionem Flori correspondent aux leçons de l'ex Ambr. in Apost., ses leçons ante correctionem rejoignent en revanche celles du PARIS n.a.l. 1437 (non corrigé ou ante correctionem). En voici quelques exemples :

F $\quad$ codex FiRENZE Plut. XIV. 21

P $\quad$ codex PARIS n.a.l. 1437

AMBr. texte d'AMBr. in psalm. 118 serm. (éd. CSEL 62)

Ambr. compilation Flor. LvGD. ex Ambr. in Apost. (éd. CCCM 193A)

- AMBR. 3,22 (p. 53,22 : F, f. 20r,4 ; P, p. 39,b,26)

ingrauescit $\mathbf{F}^{\text {a.c. }} \mathbf{P}$

ingrauescat $\mathbf{F}^{\text {p.c.Fl. }}$ Ambr. (p. 141,197,9)

- AMBr. 4,6 (p. 70,24 : F, f. 26v,4 ; P, p. 53,b,15)

clauditur $\mathbf{F}^{\text {a.c. }} \mathbf{P}$

claudimur $\mathbf{F}^{\text {p.c.Fl. }} A m b r$. (p. 27,33,3)

- ${ }^{668}$ AMBR. 4,24 (p. 79,5: F, f. 29v,13; P, p. 61,a,5)

conditorem $\mathbf{F}^{\text {a.c. }} \mathbf{P}$

cognitorem $\mathbf{F}^{\text {p.c.Fl. }} A m b r$. (p. 10,7,4)

- AMBr. 7,9 (p. 132,10 : F, f. 47v,13 ; P, p. 106,b,16)

pietate est F $\mathbf{P}$

non adquiescit sup.lin.add. $\mathbf{F}^{F l}$ add. Ambr. (p. 240,343,4-5)

- AMBr. 10,43 (p. 229,19 : F, f. 78r,25 ; P, p. 183,b,4)

nunc titubat $\mathbf{F}^{\text {a.c. }} \mathbf{P}$

nec titubat $\mathbf{F}^{\text {p.c.Fl. }}$ Ambr. (p. $\left.133,187,16\right)$ 
P. Chambert-Protat, « Deux témoins d'Ambroise sur le Psaume 118 et leur ancêtre », 2017

DOI 10.1484/M.USML-EB.5.115037; halshs-01631460

- AMBr. 14,41 (p. 326,25 : F, f. 108r,26 ; P, p. 264,b,14)

discriptio $\mathbf{F}^{\text {a.c. }} \mathbf{P}^{\text {a.c. }}$ descriptio $\mathbf{P}^{\text {p.c. }}$

adscriptio $\mathbf{F}^{\text {p..Fl. }} A m b r$. (p. 29,37,16)

- AMBr. 20,2 (p. 445,21 : F, f. 142r,26; P, p. 361,a,13)

quod inlesa ferantur ... reparare $\mathbf{F}^{\text {a.c. }} \mathbf{P}^{\text {a.c. }}$ inlisa $\mathbf{P}^{\text {p.c. }}$

quo inleso feruntur ... reparari $\mathbf{F}^{\text {p.c.Fl. }}$ Ambr. (p. 215,305,4)

Ces quelques exemples suffisent à conclure. Le codex de Cluny, PARIS n.a.l. 1437, ne descend pas du second exemplaire de Florus, FIRENZE Plut. XIV. 21. Mais il a été copié directement sur le premier exemplaire de Florus, désormais perdu ${ }^{33}$ : exemplaire que je propose d'appeler $\Phi$, par allusion aussi bien à Paris qu'à Firenze, et parce que ce sigle n'est pas encore utilisé dans la tradition manuscrite des œuvres d'Ambroise.

\section{Conclusions}

Cette phylogenèse des manuscrits « floriens » d'AMBR. in psalm. 118 serm. (Fig. ci-dessous) ouvre à son tour de nouvelles pistes de réflexion.

Pour la recherche florienne, d'abord, elle pose un «avant» et un «après », dans la chronologie relative des activités de Florus. Par exemple, dom Charlier a signalé dans PARIS n.a.l. 1437 le sigle D'F' de Florus, correspondant à $\stackrel{\underline{6} 69}{l a}$ préparation de l'extrait FLOR. LVGD. fid. 3,51. On retrouve le même sigle dans Plut. XIV. 21, de la main du copiste. Les extraits d'AMBR. in psalm. 118 serm. de

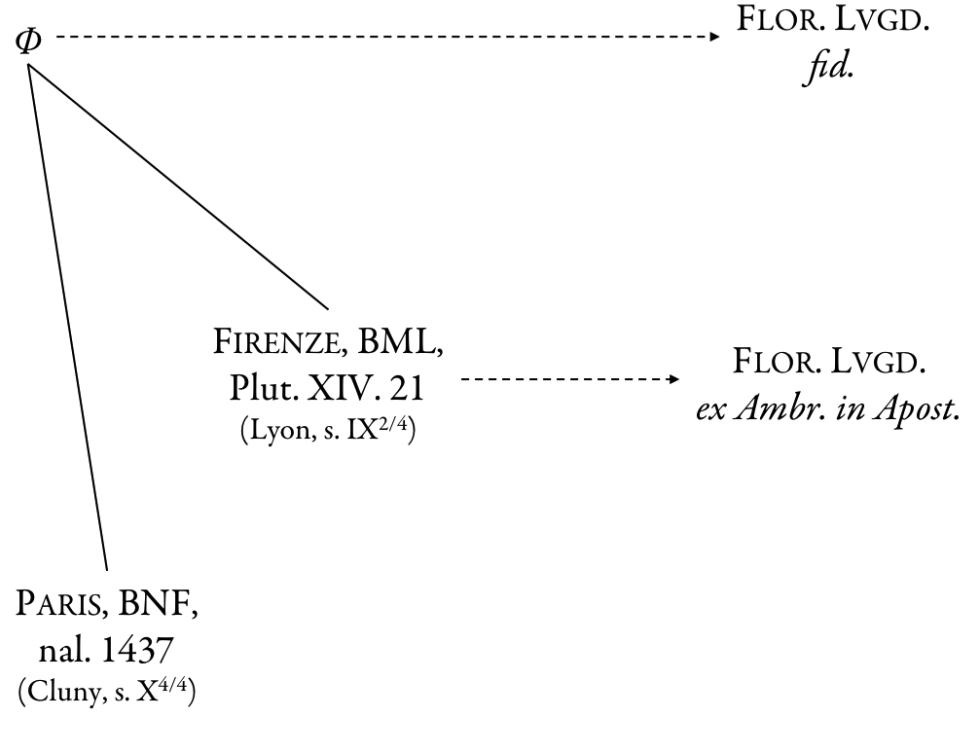
FLOR. LVGD. fid. ont donc été préparés sur $\Phi$. Inversement, on a vu que les extraits d'AMBR. in

33. Le codex $\Phi$ aurait à bon droit été qualifié de vetustissimus, mot qu'employa Anselme Le Michel pour désigner l'un des deux exemplaires d'AMBR. in psalm. 118 serm. qu'il vit à Cluny dans les années 1640 (cf. supra). Si l'on suppose, avec Veronika von Büren, que ce «Vetustissimum » de Cluny était le modèle du PARIS n.a.l. 1437, alors $\Phi$ aurait survécu jusqu'au milieu du XVII ${ }^{\mathrm{e}}$ siècle et ne se serait perdu que depuis lors. Mais en l'état des recherches, cette hypothèse reste complètement invérifiable. 
P. Chambert-Protat, « Deux témoins d'Ambroise sur le Psaume 118 et leur ancêtre », 2017 DOI 10.1484/M.USML-EB.5.115037; halshs-01631460

psalm. 118 serm. de FloR. LVGD. ex Ambr. in Apost. ont été préparés sur le Plut. XIV. 21. Des preuves matérielles viennent ainsi confirmer les déductions de Charlier sur l'antériorité de la collection De fide, probablement une ouvre de jeunesse de Florus. Cela dit, étant donnée la période d'activité de Florus (ca. 825-855), la confection du Plut. XIV. 21 doit plutôt être fixée au second quart du IX siècle, et non au premier comme l'estimait Bischoff.

On a aussi remarqué une évolution dans la méthode des sigles scripturaires : ceux qu'a copiés le copiste de Plut. XIV. 21 ont d'abord la préposition (ADCOR·), puis la perdent (COR·) ; et les sigles autographes de Florus ne l'ont jamais. Or LYON, BM, 475, autre manuscrit ambrosien préparatoire de la collection De fide de Florus, juxtapose également des sigles scripturaires avec ou sans préposition. Les sigles des Évangiles ne l'ont jamais (MT'; MR'; IOH'; aucune occurrence de Luc) et ceux des épittres pauliniennes non plus (GAL'; COR $\cdot \mathrm{I} \cdot$ ) ; tandis que les autres, correspondant à des livres de l'Ancien Testament, portent encore la préposition (INSAM'; INES'; INREG'; INCANT'; INEXH'; ${ }^{670}$ INECCLS'; INNUM'), sauf une occurrence des Juges (IUDIC'). Dans les notes autographes du Plut. XIV. 21, Florus ne met plus la préposition à aucun sigle scripturaire, même pour des livres très rarement siglés (SAM'; ES'; REG'; EXH'; ECCLSS'; NUM ; APOK' ; BARUCH' ; AMOS' ; OSEE'...). Si Florus n'avait pas été contraint de travailler sur une copie de son premier exemplaire, ces sigles postérieurs se seraient retrouvés inextricablement mêlés aux sigles antérieurs, et il aurait été extrêmement difficile de les dater relativement les uns aux autres. Mais la copie de $\Phi$ dans le Plut. XIV. 21 permet de présumer que l'idée d'abandonner la préposition — c'est-à-dire d'étendre le système des sigles évangéliques aux épîtres pauliniennes d'abord, puis à tous les autres livres - est venue à Florus graduellement, au cours des lectures qu'il avait entreprises pour sa collection De fide. On n'en était pas encore à collecter des centaines d'extraits sur l'Apôtre ; mais ce saut méthodologique de la siglaison, en systématisant le balisage scripturaire des textes patristiques, était un préalable intellectuellement important dans la genèse de cette vaste entreprise.

Plus généralement, on se demande pour quelles raisons l'événement lui-même a eu lieu. Florus avait déjà bien travaillé sur $\Phi$; quand soudain il en fait prendre une copie, et ne travaille plus désormais que sur elle. Il a probablement demandé au copiste de copier non seulement le texte d'Ambroise, mais aussi minutieusement son propre paratexte (ses notes, ses sigles, ses signes disséminés dans le texte), comme pour « sauvegarder » son travail personnel sur Ambroise. Pourtant le copiste choisi n'était peut-être pas le plus qualifié pour ce travail, puisqu'il ne 
P. Chambert-Protat, « Deux témoins d'Ambroise sur le Psaume 118 et leur ancêtre », 2017

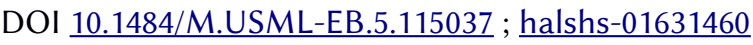

comprenait pas bien le sens de ces sigles et signes. Dans de telles conditions, des erreurs devaient inévitablement se produire : et cela n'a pas manqué, comme en attestent les corrections que Florus a apportées ensuite au corpus de notes copiées par le copiste. Quelle nécessité y avait-il donc à tout cela ? Si quelqu'un, apprenant que Florus possédait le commentaire d'Ambroise sur le psaume 118, lui en avait demandé un exemplaire, sans doute Lyon aurait envoyé une copie. Mais si Florus a gardé la copie, c'est sans doute parce qu'il ne pouvait pas faire autrement que d'envoyer l'original. Cela implique que, d'une part, Florus ne pouvait pas refuser, eu égard à la qualité du demandeur ; que d'autre part, l'exemplaire avait un certain caractère qui le rendait irremplaçable aux yeux du demandeur.

Si $\Phi$ était venu à Lyon dans le cadre d'un prêt, on aurait sans doute commencé par en prendre une copie, et Florus n'aurait travaillé que sur elle, laissant le codex original repartir chez son propriétaire. Inversement, si Florus a pris la liberté et le temps d'entreprendre certains travaux directement sur $\Phi, \stackrel{671}{=} c^{\prime}$ est qu'il ne s'attendait certainement pas à voir partir le codex. La nécessité de se dessaisir de «son» exemplaire de travail personnel dut être pour lui une désagréable surprise. Par conséquent, quelle qu'ait été son origine par ailleurs, au moment où Florus l'a connu $\Phi$ se trouvait à la cathédrale de Lyon non pas dans le cadre d'un simple prêt, mais bien comme sa propriété ; et lorsque $\Phi$ a quitté Lyon, c'était pour changer de propriétaire. Quelle personne ou quelle institution pouvait avoir des arguments suffisamment puissants pour qu'un célèbre érudit renonce à son exemplaire de travail, pour que la bibliothèque capitulaire d'une Église primatiale et métropolitaine cède un de ses trésors ?

Quant aux caractères qui distinguaient cet exemplaire particulier, on ne peut les déterminer qu'en traquant les indices qui, à travers les deux copies, trahissent l'apparence de $\Phi$. C'était un manuscrit en onciale ou semi-onciale à deux colonnes, comme la copie clunisienne - alors que la copie lyonnaise est à longues lignes, comme tous les manuscrits patristiques lyonnais de cette époque. Étant donné le volume de texte du commentaire ambrosien, cela devait faire un codex monumental. Certains détails graphiques, en particulier les titres initiaux et finaux et leurs tildes

$\mathrm{P}$

$M(\mathrm{~B}$

A abréviatifs, évoquent également des manuscrits pré-carolingiens. En particulier un élément curieux, que le copiste lyonnais du Plut. XIV. 21 a reproduit après le titre final, permettrait peut-être d'orienter les recherches 
P. Chambert-Protat, « Deux témoins d'Ambroise sur le Psaume 118 et leur ancêtre », 2017

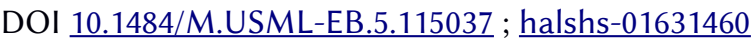

sur l'origine de $\Phi$ : une invocation « Ora pro me » portée sous la forme d'une croix grecque (Fig. ci-contre) $)^{34}$. Je n'ai pas encore trouvé d'autre attestation de ce symbole.

Aux yeux des érudits carolingiens, la valeur unique de $\Phi$ devait tenir à son antiquité, et peut-être à son origine géographique. Lyon possédait une belle collection de manuscrits tardoantiques et pré-carolingiens venus d'Italie. Florus lui-même a, par exemple, annoté un Hilaire in Psalmos italien du $\mathrm{V}^{\mathrm{e}}$ siècle, en onciale, dont il ne reste qu'un quaternion (PARIS, BNF, lat. 152, f. 9-16). Inversement, l'Expositio augustinienne de Florus sur l'Apôtre a rapidement $\stackrel{672}{=}$ passé les Alpes après sa publication dans les années 850, puisque l'exemplaire BRESCIA, Bibl. Civ. Queriniana, G. III. 2, a été copié vraisemblablement à Milan, au troisième quart du $\mathrm{IX}^{\mathrm{e}}$ siècle ${ }^{35}$. Ne peut-on penser que des manuscrits originaires d'Italie du Nord ont pu atteindre le cœur de l'Empire carolingien via Lyon, clef des routes des Alpes et de la Méditerranée ?

Quoi qu'il en soit, les relations de PARIS, BNF, n.a.l. 1437 (Cluny, dernier quart du $\mathrm{X}^{\mathrm{e}}$ siècle) et de FIRENZE, BML, Plut. XIV. 21 (Lyon, second quart du IX siècle) doivent être prises en compte pour l'histoire et l'établissement du texte du commentaire d'Ambroise sur le psaume 118. Le codex de Lyon en est le plus ancien témoin complet conservé. Le codex de Cluny n’a perdu qu'un seul feuillet. Et ils offrent deux visions d'un même codex plus ancien, $\Phi$, que l'érudition carolingienne tenait en haute estime et sur lequel Florus n'a pas eu le temps de beaucoup intervenir avant de s'en défaire. Ces circonstances rares doivent être étudiées plus en détail, afin d'établir plus précisément la physionomie du codex $\Phi$, mais aussi l'envergure et la nature exacte des interventions de Florus sur le texte d'Ambroise. On saisira ainsi à la fois le texte hérité de l'antique, et l'apport propre de l'érudit carolingien à sa tradition.

Pour conclure, ce cas illustre bien la quantité et la qualité des informations qu'on peut tirer d'une analyse fine du phénomène de l'annotation marginale. Plus que le texte annoté, le corpus de notes conserve une stratification des lecteurs et des lectures des exemplaires. Reconstituer cette stratification permet de marquer les étapes de la transmission d'un texte et de sa réception auprès des lecteurs qui, au long des siècles, se sont interrogés avant nous sur les mêmes passages.

34. Je remercie beaucoup David Ganz d'avoir décrypté la formule.

35. BisCHOFF, Katalog, t. 1, nº 683. 